\title{
Chronische myeloische Leukämie Nilotinib: besserer Schutz vor Progression und gut verträglich
}

\author{
Nilotinib könnte sich zum neuen Standard in der Therapie der \\ Chronischen Myeolischen Leukämie (CML) entwickeln. In den \\ 24-Monats-Daten der ENEST-nd-Studie (Evaluating Nilotinib Efficacy \\ and Safety in Clinical Trials of Newly Diagnosed Ph+ CML Patients) \\ schützte es im Vergleich zu Imatinib besser vor Progression, war \\ hinsichtlich der MMR-Raten überlegen und besser verträglich.
}

Das wesentliche Risiko für $\mathrm{Pa}$ tienten mit neu diagnostizierter CML besteht in der Progression, erklärte Tim H. Brümmendorf, Aachen. Seit in der CML-Therapie Tyrosinkinaseinhibitoren, wie Imatinib und Nilotinib, eingesetzt werden, unterscheiden sich die Gesamtüberlebensraten dieser Patienten kaum noch von denen der Gesamtbevölkerung. Dieses Ziel ist aber nur zu errei- chen, wenn die Progression der CML möglichst früh im Krankheitsverlauf gebremst wird und dieser Effekt langfristig erhalten bleibt, erklärte Brümmendorf. Die 24-Monats-Daten der ENEST-ndStudie zeigten, dass Nilotinib dem bisherigen Standard Imatinib in mehreren Punkten überlegen ist [Lancet Oncol. 2011;12(9):841-51]: Die Patienten sprachen tiefer und schneller auf Nilotinib an (CCyR,
MMR, CMR), waren signifikant besser vor einer Progression in der akzelerierten Phase bzw. Blastenkrise geschützt und es gab weniger CML-bedingte Todesfälle. Zudem wurde Nilotinib besser vertragen, eignet sich somit auch für die chronische Langzeittherapie. Angesichts der minimalen Kreuzunverträglichkeit zu Imatinib könnte ein Wechsel auf Nilotinib für Patienten Vorteile bringen, die eine Imatinib-Unverträglichkeit aufweisen oder ihre Therapieziele unter Imatinib nicht erreichen, schloss Brümmendorf. ay

Symposium im Rahmen der DGHOKongresses am 2. Oktober 2011 in Basel, Schweiz; Veranstalter: Novartis

\section{Metastasiertes Mammakarzinom \\ Hemmung der Mikrotubuli-Synthese verlängert das Gesamtüberleben}

\section{Bei lokal fortgeschrittenem oder metastasiertem Mam- makarzinom bessert eine Therapie mit dem Mikrotubuli- Synthesehemmer Eribulin das Gesamtüberleben (OS), das progressionsfreie Überleben, den klinischen Benefit und die Progressionsrate.}

In der EMBRACE-Studie wurde die Wirkung von Eribulin (Halaven, $1,23 \mathrm{mg} / \mathrm{m}^{2}$ i.v. an den Tagen $1+8, \mathrm{q} 3 \mathrm{w})$ als Drittlinientherapie $(\mathrm{n}=508)$ mit einer Therapie nach Wahl des Arztes (TPC, $\mathrm{n}=254$ ) bei lokal fortgeschrittenem oder metastasiertem Mammakarzinom verglichen. Alle Patientinnen hatten im Median vier Chemotherapien inklusive eines Anthrazyklins und eines Taxans erhalten. „In dieser stark vorbehandelten und ungünstigen Gruppe hat Eribulin als Monotherapie das Überleben nach ei- ner aktualisierten Analyse von 10,5 auf 13,2 Monate signifikant und klinisch relevant verbessert", so Andreas Schneeweiss, Heidelberg. Als wichtigste sekundäre Endpunkte besserten sich unter Eribulin die Ansprechrate (ORR) von fünf auf $12 \%$, der klinische Benefit (CBR) von 17 auf $23 \%$ sowie numerisch das progressionsfreie Überleben (PFS) von 2,2 auf 3,7 Monate, ohne Zunahme schwerer unerwünschter Ereignisse. Dieser Vorteil war unabhängig von Alter, Vorbehandlung oder HER2-Status und blieb nach einer Re-Analyse selbst dann erhalten, wenn in der TPC-Gruppe eine zuvor nicht benutzte Therapie eingesetzt wurde. Keine der beobachteten Nebenwirkungen trat in höheren Altersgruppen signifikant häufiger auf. Hae 2011 in Basel, Schweiz; Veranstalter: Eisai
Kurz notiert

Genetisch bedingte Anämien: Transfusionspflichtige Kinder brauchen früh Eisenchelatoren Angeborene Anämien wie die Thalassämie oder die Sichelzellanämie erfordern von Kindesbeinen an immer wieder Bluttransfusionen. Um eine Eisenüberladung des Organismus zu vermeiden, ist begleitend frühzeitig eine Behandlung mit Eisenchelatoren angezeigt. Mit der Transfusion eines jedes Erythrozytenkonzentrats werden dem Körper rund $250 \mathrm{mg}$ Eisen zugeführt. Weil der Organismus überschüssiges Eisen nicht eigenständig eliminieren kann, ist bereits ab einer Zufuhr von 10 bis 15 Erythrozytenkonzentraten mit einer relevanten Eisenüberladung zu rechnen. Darauf machte Holger Cario, Ulm, auf einem Symposium im Rahmen der Jahrestagung der Deutschen Gesellschaft für Kinder- und Jugendmedizin (DGKJ) in Bielefeld aufmerksam. In der Wachstumsphase reagiert der Körper besonders empfindlich auf eine Eisenüberladung. Deshalb, so betonte Regine Grosse, Hamburg, sollte man bei wiederholt transfusionspflichtigen Kindern früh eine Eisenchelator-Therapie beginnen. Als Eisenchelatoren sind das subkutan und intravenös applizierbare Deferoxamin (Desferal ${ }^{\circledR}$ ) sowie die oralen Chelatoren Deferipron (Ferriprox ${ }^{\circledast}$, dreimal täglich) und Deferasirox (Exjade ${ }^{\circledR}$, einmal täglich) verfügbar. Wegen möglicher Nebenwirkungen wie Neutropenie/Agranulozytose (Deferipron) bzw. Kreatininanstieg (Deferasinox) muss die Behandlung regelmäßig überwacht werden. 